\title{
VIOLENCIA MACHISTA EN LAS AULAS DE LAS UNIVERSIDADES
}

\author{
Machist violence in the university classrooms
}

Inma Lopez Francés

Pablo Tapía

Fecha de recepción: 14/09/2018

Fecha de aceptación: 09/12/2018

RESUMEN: El presente estudio tiene como propósito aportar datos acerca de las percepciones del estudiantado universitario sobre la igualdad y la violencia machista de dos universidades: Universitat de València (UV) -España- y Universidad Autónoma del Estado de Morelos (UAEM) -México-. Se llevó a cabo una investigación plurimetodológica-cuestionario y grupos de discusión- con el fin de valorar la situación actual, estableciendo diferencias y semejanzas en función del rol de la universidad; el profesorado universitario; la formación; las intenciones; la percepción de la violencia machista y el lenguaje inclusivo. La muestra está formada por 835 estudiantes (España: 435- México: 400). Los resultados apuntan que en ambas universidades siguen existiendo elementos que generan desigualdad, discriminación y violencia machista. Estos hallazgos han de servir para tomar decisiones en el contexto universitario que permitan erradicar todo atisbo de violencia machista.

PALABRAS CLAVE: Violencia machista, Universidad, Feminismo, Educación.

ABSTRACT: The purpose of this study is to provide information about the perceptions of University students on equality and gender violence at two universities: Universitat de València (UV) -Spain- and Universidad Autónoma del Estado de Morelos (UAEM) -México-. A multimethodologic investigation-questionnaire and discussion groups-was conducted in order to assess the current situation, establishing differences and similarities depending on the role of the university; the university teaching staff; formation; intentions; perception of sexism violence and inclusive language. The sample consists of 835 students (Spain: 435- Mexico: 400). The results indicate that in both universities there are still elements that generate inequality, discrimination and sexist violence. These findings should be used to make decisions in the university context to eradicate all signs of sexist violence.

KEY WORDS: Gender violence, University, Feminism, Education.

\section{Introducción}

Nuestra pesquisa parte de una perspectiva crítica y feminista. El feminismo no es una teoría más. Es una conciencia crítica que resalta las tensiones y contradicciones que encierran los discursos. Ése es el espíritu: una teoría de la justicia que ha ido cambiando el mundo. Es la linterna que muestra las sombras de todas la grandes ideas gestadas y desarrolladas sin las mujeres y en ocasiones a costa de ellas: democracia, desarrollo económico, bienestar, justicia, familia, religión... Su luz es la justicia que ilumina las habitaciones oscurecidas por la intolerancia, los 
prejuicios y los abusos (Varela, 2005). Por ello, nos ponemos en marcha por las silenciadas, las humilladas, vilipendiadas, ultrajadas, cosificadas, traficadas, maltratadas, acosadas, violadas, asesinadas. Por las que se mantienen en pie porque las posibilidades de su contexto lo permiten; por las que aun desprovistas de todo apoyo también lo hacen; por las que callan porque han de decidir entre el silencio o la muerte; por las sexualizadas, racializadas, generizadas y sexuadas; por las mujeres transexuales y las cissexuales; por las que se mantuvieron en pie de guerra y resistieron, por aquellas que lo hacemos ahora y por las generaciones que vendrán; por todas ellas, pues la lacra que a todas ellas asedia es responsabilidad de todos, todas y todes.

Y es que en pleno siglo XXI existen elementos estructurales que oprimen a las mujeres, impidiendo que se desarrollen en igualdad de condiciones. En nuestra investigación pretendemos indagar si del mismo modo que ocurre en el resto de ámbitos sociales y culturales, en la Universidad también hay situaciones discriminatorias hacia las mujeres, mostrando la problemática de la violencia machista como una cuestión que no conoce fronteras y que afecta a la convivencia en dicho espacio. El objetivo de aprender a convivir forma parte, al menos de forma implícita, de todo proceso educativo. El Informe Delors (DELORS, 1996), resalta cuatro pilares como esenciales para la educación del siglo XXI: aprender a conocer, aprender a hacer, aprender a ser y aprender a convivir. Y alude a este último, el aprender a vivir, como la utopía que es prioritaria alcanzar. En este aprender a convivir no se busca únicamente transmitir unas determinadas estrategias y habilidades para resolver conflictos, sino que se quiere construir una nueva cultura y relaciones sociales donde la violencia no tenga cabida. Bajo todo esto nos preguntamos ¿Las Universidades son espacios libres de violencia? ¿Son espacios donde las personas están aprendiendo teórica y prácticamente a convivir?

\section{Violencia machista, ¿un conflicto mediable?}

El presente artículo forma parte del monográfico que lleva por título Convivencia, resolución de conflictos y mediación, por ello, también va a intentar responder a la pregunta ¿es la violencia machista una cuestión mediable? Una definición clásica acerca del concepto de conflicto es la ofrecida por De Bono (1990) como "choque de intereses, valores, acciones o direcciones. Se aplica la palabra conflicto, desde el momento en que este choque tiene lugar, incluso cuando haya un conflicto potencial, esto significa que ya hay un conflicto de dirección, aunque el choque aún no se haya producido" (p.45). Tradicionalmente, el conflicto ha ido cargado de connotaciones negativas. Viana (2012) afirma que, de forma general, en las culturas occidentales predomina una concepción negativa del conflicto derivada de la ideología tecnocrática conservadora, que lo presenta como algo negativo, no deseable. Estamos de acuerdo en que se ha de descubrir la perspectiva positiva del conflicto, considerándolo como algo consustancial al ser humano, inherente a las organizaciones e incluso deseable y necesario para el avance y el desarrollo tanto personal como social. En este sentido, el uso de la mediación en el tratamiento de conflictos es el camino hacia la erradicación de la exclusión como forma de relación social.

Sin embargo, ¿Podemos situar la violencia machista? ¿Es un conflicto de intereses entre hombres y mujeres? ¿Se trata tan solo de valores contrapuestos? ¿Una lucha ideológica? ¿Una disputa de poderes? ¿O se trata de algo más? En sí, la violencia machista es interseccional, abarca numerosas esferas de la vida política, económica, profesional, social y personal; tanto en el ámbito de lo público como de lo privado. 
"La diversidad de experiencias de discriminación en que se inscriben las mujeres puede y debe complementarse con un análisis teórico general, cuya génesis se encuentra en la propia existencia empíricamente contrastable de que existe una estructura de dominación y hegemonía masculina en todas las sociedades" (Cobo, 2009, p.51).

Por ende, no se trata de problemas aislados, diferenciados radicalmente entre sí y propios de una o varias relaciones interpersonales que se dan en el marco de un contexto concreto, unos sujetos y unos procesos propios, privados, puntuales y experienciales derivados del devenir de una(s) relación(es) que se ve(n) deteriorada(s) por el tiempo o las circunstancias vitales de quiénes la forman, sino que la violencia machista responde a una estructura universal, inscrita y presente en todo el Planeta Tierra, que se mantiene más allá de las diferencias sustanciales de cada momento, relación y en todas las formas sociales.

Además, Bell y Smith (2001) indican que un conflicto mal gestionado puede llevar al bloqueo en la comunicación, restringiendo informaciones importantes, distorsión deliberada de los hechos para defender una posición rompiendo la coalición de grupo y formando emparejamientos o subgrupos (...) propiciando de este modo una lucha de ganadores-perdedores casi imposible de resolver. Algo que ni si quiera se acerca a las verdaderas consecuencias de la violencia machista, explicitadas por el equipo de Mujeres Para la Salud (2018):

La conducta violenta, tanto en el terreno físico como en el psíquico, va causando un deterioro (...) en la mujer que (...) se manifiesta en una auténtica sumisión a los deseos y órdenes del agresor. El agresor llega a tener un control y dominio de la mujer que hace que cada vez sea más inflexible, por lo cual la violencia continúa su ciclo de intensidad creciente, y la mujer llega a perder su propia identidad para convertirse en una posesión más (sp).

Las distintas configuraciones de violencia machista son numerosas, si bien no son percibidas con una gravedad homogénea. Puesto que, a pesar de los esfuerzos aparentes a nivel estatal en sensibilizar a la sociedad acerca de la violencia machista y sus características, parece ser que no es suficiente cuando en una encuesta realizada por el Ministerio de Sanidad, Servicios Sociales e Igualdad (2015) tan solo un $44 \%$ de hombres de entre 15 y 29 años perciben la violencia machista como extendida (frente a un 63\% de mujeres), ambos coincidiendo en que este tipo de violencia específica se proyecta, sobre todo, en "los salarios" y "la posibilidad de compaginar vida laboral y familiar". Además, el mismo estudio recoge que no todas las formas de violencia suscitan el mismo rechazo, en concreto, aquellas formas de violencia de control, suscitan polaridades. Mientras que un 67\% la rechaza, un 33\% percibe como aceptables actitudes tales como "controlar los horarios de la pareja" o decirle a ésta "cosas que puede o no puede hacer".

Así pues, con todo lo expuesto anteriormente, se puede afirmar que la violencia machista no es compatible con la definición de conflicto dado que su circunstancia y epistemología no concuerdan con la de este concepto. Además, tampoco las consecuencias de una mala gestión de conflictos y de la violencia machista presentan ninguna aproximación o similitud significativa. Sumado, finamente, a la especificidad que aporta Núñez a este tipo de violencia concreta. Afirmamos, por tanto, que la violencia machista ni es un conflicto ni es mediable.

1.1.Violencia machista, ¿qué es entonces? 
Como recogen diversas autoras (hooks, b. 1990; Butler, J. 1990; Varela, N. 2005; Wittig, 2006;Solà, M. y Urko, E. 2013; Goldman, E. 2017) la violencia machista se articula en y desde un aparato ideológico que comprende valores, creencias y acciones, traducidas en roles asociados a características diversas que pueden variar en función del sistema político, económico, social y cultural (normalmente, al aparato sexo-género; sexo biológico asignado al nacer que se vincula intrínsecamente con el género, algo que varias y varios autores negarían bajo el espectro de la Teoría Queer), donde la figura del varón se postula como suprema, absoluta y contenedora de todo poder construyéndose a sí mismo como sujeto activo político y tornando a todo aquello que, bajo su cosmovisión, no es natural, activo o sujeto, en antinatural, pasivo y objeto; en este caso la figura de la mujer.

Todo ello se establece y posibilita a través de configuraciones donde se sitúa a la figura del hombre en el centro (androcentrismo) sociopolítico y económico de todo, situando a las mujeres en una posición desprovista de todo poder y reconocimiento, identidad, otredad y dignidad, vulnerando de forma severa sus Derechos Humanos. En una palabra: hablamos de patriarcado. O al menos de eso se habló, especialmente, hasta las décadas de los 60 y los 70 (De Miguel, 2007). A lo largo de los años posteriores, las investigaciones y conceptualizaciones en torno a los feminismos, que han proliferado tímidamente y ahondado más en la cuestión en sí, en la raíz; la incorporación de las tecnologías de la información y la comunicación a la lucha feminista (entre otros factores) han permitido una rápida evolución y ampliación de los imaginarios feministas, su alcance e impacto.

Así, al patriarcado se le suma un prefijo cargado de significancia: hetero-. Pese a que Fontanela (2008) realizara una brillante labor en su definición de patriarcado explicitando su alcance público y privado, interclasista e intragénero, individual y colectivo, así como de sus medios pacíficos o violentos, la complementación que realiza Silva (2015) es impecable:

"[el heteropatriarcado] se basa en la aceptación de un único modelo de familia universal, compuesto tradicionalmente por una pareja heterosexual (...) así como en la perpetuación, reparto y legitimación social de una serie de roles para hombre y mujer. (...)el poder de dominación del hombre sobre la mujer desde la heterosexualidad, o si se quiere, desde la heteronormatividad, excluyendo otro tipo de relaciones afectivosexuales" (sp).

En suma, la violencia machista, (hetero)patriarcal o de género se encuentra apoyada y legitimada por un sistema que crea y proyecta la división social basada en los roles de género (sujetos a su vez a un sistema dicotómico sexo-género) y su derivada desigualdad y violencia múltiple proyectada a través y desde los parámetros previamente mentados hacia las mujeres. Podríamos afirmar que esta colosa problemática es una cuestión de datos (OIG de América Latina y el Caribe, 2015; ONU MUJERES, 2017):

1. El $35 \%$ de las mujeres de todo el mundo han sufrido violencia física o sexual por parte de su compañero sentimental o violencia sexual por parte de una persona distinta a su compañero en algún momento de su vida.

2. Aquellas que han sido víctimas de violencia física y/o sexual tienen más del doble de posibilidades de tener un aborto, casi el doble de sufrir depresión (entre otros) y, en determinadas regiones, 1'5 veces más posibilidades de contraer el VIH. 
3. En prácticamente la mitad de los casos de mujeres víctimas de homicidios en el mundo en 2012, el autor fue un familiar o compañero sentimental, frente a menos del $6 \%$ de hombres asesinados en ese mismo año.

4. En 2014, en 25 países de América Latina y el Caribe un total de 2089 mujeres fueron víctimas de feminicidio. El feminicidio comprende un vasto conjunto de situaciones: muertes provocadas por mutilación, violación, palizas, persecuciones; muerte de brujas en Europa, las inmolaciones de novias y viudas en la India y los crímenes de honor en algunos países de América Latina y Oriente Medio.

5. Más de 750 millones de mujeres que viven actualmente en todo el mundo han vivido un matrimonio infantil forzado, algo que suele dar lugar a embarazos precoces no deseados, al aislamiento socioafectivo, la interrupción de la escolarización y la propensión a sufrir violencia física y psicológica.

6. Al menos 200 millones de mujeres y niñas que viven actualmente han sufrido la mutilación genital femenina.

7. Las mujeres y niñas representan el $71 \%$ de víctimas de trata de personas, casi tres de cada cuatro con fines de explotación sexual.

8. 1 de cada 10 mujeres de la Unión Europea declara haber sufrido ciberacoso desde los 15 años. Incluye: correos electrónicos o mensajes SMS no deseados, sexualmente explícitos y ofensivos, así como intentos inapropiados y ofensivos en redes sociales.

9. En, al menos, 27 universidades de los Estados Unidos en 2015, el 23\% de las estudiantes universitarias de pregrado declararon haber sufrido algún tipo de agresión o conducta sexual indebida.

10. En 39 países de cinco regiones distintas, el $82 \%$ de las parlamentarias encuestadas declararon haber experimentado alguna forma de violencia psicológica durante su mandato (comentarios, gestos e imágenes de índole sexual, [machistas] o humillantes, amenazas y acoso.

Aunque los datos no lo son todo. Recordemos: son una pequeña muestra de la realidad. Existen muchos países y regiones que se niegan a ofrecer los datos acerca de la violencia machista y los que han posibilitado recogerlos no representan una muestra total de las tantas y tantas realidades de mujeres diversas alrededor del globo (ONU MUJERES, 2017). No es una simple cuestión de datos; es una cuestión de justicia, bienestar social y convivencia.

\section{Violencia de género en las universidades}

Tal y como se menciona en el apartado anterior, la violencia machista es una cuestión interseccional que abarca el total espectro de nuestra vida y nuestras vivencias. Podemos verlo quizá más claramente tras los numerosos datos aportados por los organismos supranacionales: hablamos de economía, sanidad, seguridad, política, derechos y educación, entre otros.

Se centrará ahora el foco de atención en esta última; más específicamente en la educación superior universitaria. No obstante, con tal de que el estudio de este ámbito y cómo podría afectar o no al combate feminista del heteropatriarcado tenga sentido, debemos plantearnos si el objetivo principal de la universidad es la reproducción del orden social existente o la transformación social.La institución ha sufrido cambios a lo largo del tiempo, siendo un espacio diseñado por y para las élites en sus primeras configuraciones del siglo XX, pasando por una apertura a las clases trabajadoras en la segunda mitad y a una posterior masificación. (Luxán Serrano, M., Imaz Bengotxea, J., Bereziartua Etxeberria, G., y Lauzurika Arrondo, A., 2014). ¿Y ahora? ¿De qué tipo 
de universidades hablamos en el siglo XXI? En palabras de Cadavid G., T. E. (2009) hablamos de un enfoque empresarial y capitalista de la universidad, del tipo "empresa de servicios", en la que la calidad educativa y formativa es una sombra situada en la periferia de la importancia mientras que el interés económico se sitúa en el centro, constituyendo así lo que debería ser un espacio didáctico, de formación, aprendizaje y experiencia, en un ente administrativo, tornando el saber y la vivencia en bienes de consumo y mercancías, sujeta a las leyes del mercado y su lógica utilitarista. No es de extrañar, por tanto, que la esencia que vertebra las instituciones universitarias copien y reproduzcan los modelos socioeconómicos de las sociedades a las que están sujetos: machistas y patriarcales.

Encontramos así en el contexto del Estado Español numerosas investigaciones que indagan en este reflejo mercantilista-heteropatriarcal, que se traduce en nuestras universidades en violencia machista. En el estudio llevado a cabo por Ferrer, Ramis, Torres y Navarro (2006) realizado con 1395 estudiantas y estudiantes universitarios de las Islas Baleares se revela que subsisten creencias erradas (la víctima es culpable en determinadas circunstancias de que la golpeen o de que sufra asalto sexual) que conllevan ciertas actitudes que toleran la violencia machista. Reconocen que es un factor de riesgo que hay que prevenir, siendo un indicador clave para la prevención haber recibido formación específica en la temática, puesto que el estudiantado muestra actitudes menos favorables hacia la violencia al haberla recibido, como también hallan Macías y otros (2012).

Valls, Flecha y Melgar (2008) muestran en su su estudio realizado las Universidades de Barcelona, Lleida, Rovira i Virgili y la Universitat Nacional d'Educació a Distància que una de las principales dificultades para la erradicación de la violencia machista es que no se reconocen algunas de las situaciones que se definen como violencia machista ya que, de forma general, el $14 \%$ de las encuestadas afirmaba haber sufrido violencia machista en el ámbito universitario y, al preguntar manifestaciones concretas, un $44 \%$ admitió vivirlas. Además, la investigación anota que en los ambientes académicos (entrevistas, momento de la encuesta) las chicas tenían reparo en admitir ser víctimas de violencia, en situaciones de "íntimas" lo revelaban con mayor facilidad.

González y Mora (2014) expone que las mujeres de la Universitat de València también sufren violencia machista. Con una muestra de 3404 personas procedentes del Personal de Administración y Servicios, Personal Docente e Investigador y estudiantado, se revela que el $36{ }^{\prime} 05 \%$ han sufrido o sido testigos en su entorno más próximo de una situación de violencia machista, suponiendo un 20'03\% ocurrido dentro de la universidad. Algunos datos a destacar son: 1) un $62^{\prime} 02 \%$ tuvieron problemas de salud psicológica; 2) un $37^{\prime} 09 \%$ repercusiones en el entorno laboral; 3) aislamiento social y problemas de salud física entre un $29 \%$ y un 22\%; 3) repercusiones económicas y cambios de lugar de residencia alrededor de un $12 \%$.

Estas son solo algunas de las investigaciones realizadas en el contexto del Estado Español. Recordemos que no solo se trata de datos; estos datos recogen vivencias, en algunas ocasiones insoportables y explícitas, en otras soportadas largo tiempo e implícitas, pero siempre injustas y altamente vejatorias. Como podemos observar, las universidades españolas parecen no ser un espacio seguro (tampoco) para ellas. Algo que, como se demuestra a continuación, no es un caso aislado que solo ocurra en este país.

En México, más concretamente en la Universidad de Guadalajara, también ocurre. Con detalle, Enciso y Rico (2009) muestran en su investigación que existe una aparente igualdad, en consonancia con la normativa, que oculta formas de discriminación vinculadas a los esquemas 
tradicionales asignados a cada género. A pesar de los esfuerzos a nivel normativo, ponen de relieve la existencia de una cultura institucional que implica, de forma muy integrada y naturalizada, una práctica machista, misógina y homófoba que genera diversos fenómenos, difícilmente reconocibles por ser considerados naturales, que provocan inequidad de género.

Palomar $(2009,2011)$ enfatiza la necesidad de conocer y visibilizar la cultura institucional de las universidades, alumbrando los cimientos sobre los que se establecen relaciones y reglas institucionales que reproducen las prácticas sociales, planteándose algunas preguntas como: ¿cuáles son las normas, escritas o no, que cumplen una función reguladora de las relaciones de género en la vida comunitaria?, ¿cómo se expresa y se produce el género en el lenguaje y estilo de comunicación propios de la Universidad, como parte de su identidad?, ¿cómo se construye y se refleja el género en los materiales que produce la comunidad universitaria y que presenta la imagen institucional?, ¿cuáles son las ideas implícitas o creencias subyacentes sobre las personas y la institución?, ¿qué prácticas institucionales son utilizadas para en relación con el género en la universidad (concursos de belleza, presencia de edecanes mujeres en los espacios académicos, narrativas de violencia de género, etc.)?. A partir de sus investigaciones, considera que se han logrado modificar algunos prejuicios ideológicos en referencia al género; sin embargo, también sostiene que la equidad de género es un problema en el campo universitario, ya que la cultura universitaria tiene un trasfondo sexista y discriminatorio.

A través de los datos aquí expuestos, podemos aseverar que el espacio universitario no es un espacio seguro para las estudiantas universitarias, PAS y PDI femenino, lo que mengua o inutiliza por absoluto las posibilidades de estas mujeres de su desarrollo personal, social y profesional. Aunque, cabe destacar, el problema no radica únicamente en las (mal)vivencias que mujeres de todo el mundo viven en el espacio universitario, sino en las implicaciones proyectivas que tiene el impacto de la violencia machista interseccional en la universidad para con la sociedad, de la que forma y es, a su vez, parte.

Macías, J. et al. (2012) pone especial atención en su estudio a la formación y socialización de género:

“(...) estas creencias [machistas] están muy interiorizadas, es decir, los mecanismos a través de los cuáles se siguen transmitiendo el sexismo y la violencia son tan generales, profundos y, a veces, sutiles, que además de transmitir un modelo no sexista de relaciones en el ámbito universitario, se deben incluir en el currículum académico universitario contenidos específicos para minimizar estas creencias y mejorar la actitud ante el problema” (p.5).

Así, la universidad se postula como un agente vital en lo que refiere a la construcción, percepción y trato del género; de los géneros. Es por ello que tiene una gran responsabilidad feminista y social a la que se ha de prestar atención y evaluar; no solo a la institución como tal, sino a nosotras y nosotros mismos como agentes activos que participan de estos procesos.

Es preocupante que la violencia machista esté tan profundamente extendida y arraigada en todos nuestros ámbitos y niveles. Recordemos que la violencia machista no es solo la agresión física explícita, sino que como recogen las investigaciones anteriormente expuestas y el marco teórico anterior, se manifiestan a través de una gran y diversa cantidad de formas y colores. Así, no incluir autoras en una guía didáctica es violencia machista, por ejemplo. Debemos hacer lo 
posible por ofrecer resistencia a este fenómeno, caminar hacia una equidad justa y real; y la universidad juega un papel muy importante.

\section{Consideraciones metodológicas}

El objetivo general de la presente investigación se centra en interpretar las percepciones del estudiantado de la Universitat de València (en adelante UV) y de la Universidad Autónoma del Estado de Morelos (en adelante UAEM) hacia la igualdad y prevención de la violencia machista. Para la recolección de datos, se utilizó un cuestionario elaborado ad hoc y grupos de discusión. Con los ítems formulados, se pidió a una comisión compuesta por jueces y juezas que valoraran, con una gradación de 1 a 10, los ítems presentados en función de los siguientes criterios: claridad e idoneidad, pertinencia y consistencia. A partir de la valoración de los expertos y expertas, se realizó una prueba piloto y el resultado fue la reformulación/eliminación de varios ítems. Se utilizó el programa estadístico SPSS-19 para someter los datos a un análisis de fiabilidad y validez con el fin de lograr un cuestionario depurado. Tras los análisis recomendados, se obtuvo un cuestionario de cuarenta ítems, agrupados en cinco ejes y con una fiabilidad final de 0.833 y una varianza total explicada de aproximadamente $60 \%$. En el cuestionario se usó una escala tipo Likert de cinco opciones ( $1=$ total desacuerdo; 2=desacuerdo; $3=$ indiferente; $4=$ de acuerdo; 5=totalmente de acuerdo).

Se calcularon muestras representativas para aplicar el cuestionario con un nivel de confianza de $95 \%$ y un error de estimación de 5\%, obteniéndose un tamaño de 380.99 sujetos en la UV y 374.41 en la UAEM. Se constituyó un muestreo estratificado proporcional (por género y ámbito de conocimiento). La muestra final fue:

Tabla 1

Distribución de la muestra final. Universitat de València.

\begin{tabular}{llll}
\hline & Mujeres & Hombres & Total \\
\hline Artes y Humanidades & 45 & 19 & 64 \\
\hline Ciencias & 18 & 15 & 33 \\
\hline Ciencias de la Salud & 69 & 29 & 98 \\
\hline Ingeniería y Arquitectura & 5 & 25 & 30 \\
\hline Ciencias Sociales y Jurídicas & 117 & 93 & 210 \\
\hline TOTAL & 254 & 181 & N. 435 \\
\hline
\end{tabular}

Tabla 2

Elaboración propia

Distribución de la muestra final. Universidad Autónoma del Estado de Morelos.

\begin{tabular}{llll}
\hline & Mujeres & Hombres & Total \\
\hline Ciencias Exactas & 16 & 36 & 52 \\
\hline Ciencias Naturales & 15 & 15 & 30 \\
\hline Ciencias de la Salud & 60 & 30 & 90 \\
\hline Ciencias Sociales y Administrativas & 73 & 69 & 142 \\
\hline Ciencias Agropecuarias & 5 & 8 & 13 \\
\hline Artes y Humanidades & 37 & 36 & 73 \\
\hline TOTAL & 206 & 194 & 400 \\
\hline & & \multicolumn{2}{c}{ Nota. Elaboración propia }
\end{tabular}




\section{Análisis de los resultados}

Se realizaron los siguientes análisis: normalidad muestral de los datos, análisis descriptivos y de frecuencia, gráficos y estudio de las correlaciones entre los diferentes ítems. A partir de los resultados obtenidos se contrastaron con los testimonios recopilados en los grupos de discusión.La posibilidad de someter los datos recopilados a análisis cuantitativos y cualitativos rigurosos hace más accesible el estudio de las variables incluidas en la investigación, permitiendo operar con una mayor precisión. De este modo, se supera una de las limitaciones clásicas que se le presuponía a las investigaciones relacionadas con aspectos sociales, culturales y/o personales: su falta de rigurosidad y cientificidad.

En este artículo se expone el resultado de estos análisis cuantitativos y cualitativos, a través de la discusión de los datos más relevantes, no como una "cosecha de cifras" sino mostrando, de forma crítica y comparada las percepciones del estudiantado hacia la Igualdad y la Violencia de género en la la UV y la UAEM. La discusión se elabora a partir de la triangulación entre los cuestionarios, los grupos de discusión, las observaciones anotadas y los supuestos teóricos de la investigación. Es importante destacar que, con el fin de agilizar la lectura y el análisis, hemos combinado las cinco categorías de la escala Likert en tres: de acuerdo (valor 4 y 5), indiferente (valor 3), en desacuerdo (1 y 2).

\section{Discusión de los resultados}

En primer lugar, respecto a la función que el estudiantado le asigna a sus Universidades hacia la formación en Igualdad y prevención de la Violencia de género, comparativamente hablando, el estudiantado de la UV presenta porcentajes ligeramente más elevados de acuerdo al considerar que es función de la Universidad formar en la Igualdad entre mujeres y varones, debiendo constituirse también en un referente social (75\% en la UV frente a un 51\% en la UAEM). En los grupos de discusión de la UV una de las participantes se manifestaba en este sentido: “(...) sí es competencia [de la Universidad] por qué, aunque no se haya hecho bien antes, el proceso de aprendizaje es a lo largo de toda la vida, por lo cual la Universidad sí tiene que encargarse de ello" (M2 G1, UV). En este sentido, no ha de olvidarse tampoco que existe una parte del estudiantado que se muestra en desacuerdo al considerar que es función de la Universidad educar en el valor de la igualdad ( $13 \%$ en la UV y $25 \%$ en la UAEM), haciendo entrever que el tándem Universidad y formación en Igualdad como una cuestión de justicia y ética no es una cuestión sine qua non sino que también existen resistencias.

En el análisis también aparece la figura del profesorado universitario. La imagen presenta discrepancias entre las expectativas y la realidad percibida: un porcentaje elevado del estudiantado cree que es responsabilidad del profesorado universitario formar en Igualdad y prevención de la Violencia de género (UV: 74\% y UAEM: 69\%) sin embargo, se hallan porcentajes elevados de Indiferencia (UV: 30\% y UAEM: 28\%) y desacuerdo (UV: 20\% y UAEM: 27\%) al preguntar si creen que el profesorado universitario se preocupa por promover en sus estudiantes valores como la Igualdad y la no violencia. A colación de estas cuestiones, es interesante el siguiente testimonio: "No le dan importancia [el profesorado], por eso hacemos lo que quieren. Por ejemplo, una niña en un trabajo intentó hablar de mujeres y hombres. Por igual, ¿para qué? Otros no hicieron ese esfuerzo y luego pues no lo valoran, ese trabajo extra, entonces ¿para qué?” (H1G2, UAEM). Este es un hecho de suma importancia; si el estudiantado percibe que no se valora su trabajo en 
cuestiones relacionadas con la Igualdad y la Violencia de género es complicado que lleguen a estimarlo como un objetivo digno de asumir, dedicando sus esfuerzos hacia aquellas cuestiones que perciben que son más valoradas por parte de su profesorado que al fin y al cabo, se constituye en un referente.

En relación al profesorado, es interesante hacer hincapié en la diferencia significativa que descubre cuando se pregunta si se percibe un trato diferenciado por parte del profesorado en relación al sexo percibido. El estudiantado de la UV presenta un desacuerdo significativamente mayor (18\%) al estudiantado de la UAEM cuando se le pregunta si creen que, en su facultad, algunos profesores y profesoras tratan a su estudiantado de forma distinta. Ocurre a la inversa con el acuerdo, el estudiantado de la UAEM presenta un acuerdo significativamente mayor (19\%) que el estudiantado de la UV al responder a dicho ítem. En la UAEM recabamos testimonios que corroboran que, parte del profesorado trata de forma distinta a su estudiantado en función de su sexo: "Cuando el maestro necesita ayuda con la computadora siempre les pide a ellos, ¿cómo cree? ¿nosotras no sabemos utilizar una LAP?" (M3G1, UAEM) o "El profesor las trataba, al género femenino, muy bien. Les decía que estaban bien bonitas. Que eran muy trabajadoras. A los hombres que eran mediocres y vagos" (H2G2, UAEM). También se obtiene información en la UV a este respecto: "He llegado a ver a un profesor que llegaba a clase una chica con minifalda, así muy arreglada y le decía que se pusiera al final de la clase porque no le quería ver las piernas" (M2 G2, UV). Estos testimonios corroboran la existencia de una desigualdad de trato en función del género por parte de cierto profesorado. Esto es una cuestión de radical importancia pues su existencia, obstaculiza el desarrollo integral de las estudiantes impidiendo que manifiesten todas y cada una de sus potencialidades, vulnerando de esta forma las posibilidades de que construyan una vida digna, a la altura de sus capacidades y no encorsetada en función del género/género que se percibe.

Y ¿qué ocurre con el estudiantado? ¿qué actitudes mantiene respecto a su propia formación en Igualdad de género? En términos generales, los porcentajes revelan que los y las participantes perciben importante formarse en la Igualdad y prevención de la Violencia de género en el marco universitario (UV: $86 \%$ y UAEM: $82 \%$ ). Sin embargo, al ser más concretos aludiendo a la utilidad de introducir contenidos específicos en el currículum universitario relacionados con estas cuestiones, los porcentajes de acuerdo en ambas Universidades presentan un descenso significativo (UV: 65\% y UAEM: 54\% ). Este descenso es un hecho a tener en cuenta. En los grupos de discusión se hallan testimonios que corroboran estos datos, por ejemplo: "se deberían incluir otras áreas como el medio ambiente o la educación vial que también causan muchas muertes, que también me parecen muy importantes y nos afectan a todos. Demasiado." (V1 G2, UV) o "Yo creo que no. No, no, nada de incluir en el currículo. Se pierde tiempo de lo importante. Ya hay demasiado" (H3G1, UAEM).

Por otro lado, al abordar cuestiones relacionados con la discriminación, las desigualdades y la Violencia de género en el contexto universitario se descubre en ambas Universidades, que el estudiantado responde con porcentajes elevados en torno al valor tres: "Indiferente". Los ítems relacionados con estas cuestiones presentan matices y diferencias interuniversitarias que es necesario reseñar: el estudiantado de la UV presenta un nivel más alto de acuerdo al considerar la Violencia de género como una cuestión pública (UV: 91\% y UAEM: 78\%). Sin embargo también muestra un nivel mayor de Indiferencia (UV: $32 \%$ y UAEM: 11\%). En este sentido, se descubren aportaciones del estudiantado que no conciben la Violencia de género como un asunto público sino 
como una cuestión que han de enfrentar a quienes les afecta, en específico las mujeres: “ las mujeres lo veis siempre desde la perspectiva de víctima, no lo sé, es mi opinión. Hay medios para salir y ahí están..." (V1 G1, UV) o colectivos en riesgo de exclusión social: "la violencia de género va muy relacionado con la educación. Que normalmente los casos que vemos en la televisión son de situaciones normalmente marginales o de exclusión social, en la que, pues, hay muy poco valor a la hora de respetar a la mujer y, en ese sentido pues, no ha recibido la educación, que debería haber recibido, ese respeto hacia la mujer" (V4 G2, UV).

Por otro lado, el estudiantado de la UAEM manifiesta un acuerdo significativamente más elevado al considerar que la Violencia de género es un fenómeno que existe en el ámbito universitario aunque, también existe un porcentaje del estudiantado de la UV que reconoce su existencia (UV: 33\% y UAEM: 64\% ). En ambas Universidades se encuentran testimonios que relatan situaciones que podrían definirse como Violencia de género: "yo tenía un profesor que me daba a mi clase pero esto le ocurrió a unas compañeras que yo creo que no era acoso, ni tan grave pero sí que las llamaba al despacho, las, tenía el número de teléfono de ellas porque se lo pidió y ellas tenían miedo, porque, éste no nos va aprobar y tal.... Cosas que les daba una asignatura que era difícil, les daba las preguntas a condición de que fueran y estuvieran con él y eso..." (M5 G2, UV) o "A veces sí. Sientes agresión por el maestro. Pues molestar, insinuar, su mirada. También los compañeros" (M1 G2, UAEM). En este sentido, no hay que perder de vista que la Violencia de género que se produce en las Instituciones universitarias y entre los miembros de la comunidad es en muchas ocasiones sutil y normalizada, no siendo percibida como tal o siendo percibida como una cuestión natural o correctiva. Esta falta de reconocimiento y/o reconocimiento como una cuestión "normal" es uno de los principales obstáculos para superar y denunciar posibles prácticas abusivas que se estén dando en el seno de las Universidades. Este hecho es de suma importancia puesto que los datos, testimonios y observaciones corroboran que la Violencia de género también ocurre en las Universidades.

Relativo al uso del lenguaje inclusivo, el 34\% de la UAEM y el 18\% de la UV creen que su profesorado universitario no ve con buenos ojos la utilización de un lenguaje inclusivo. El 49\% de la UV y el 33\% de la UAEM se manifiesta en contra: el profesorado sí valora su utilización. El resto, se muestra indiferente. En los testimonios recabados se aprecia que no es tan extraño la utilización de un lenguaje sexista en las aulas: "el llenguatge que s'emprà sempre es referit a gènere masculí, el femení, de normal, no sol estar representat a no ser que és parle especificament.(...) De vegades també s'emprà un llenguatge sexista” (M3 G1, UV) o "muchas veces los maestros se meten...sí. Se quieren hacer los graciosos pero son comentarios muy machistas" (M3 G2, UAEM). Es importante resaltar que el estudiantado de la UAEM percibe en mayor medida, significativamente hablando, que el estudiantado de la UV que su profesorado universitario no ve con buenos ojos que utilicen un lenguaje inclusivo mostrando una respuesta reactiva puesto que, en mayor medida que el estudiantado de la UV, afirman la necesidad de utilizar un lenguaje inclusivo con el fin de no discriminar a las mujeres. Se halla una incoherencia ya que, a pesar de encontrar porcentajes elevados de acuerdo (UV: 70\% y UAEM: 64\%) al considerar que acabar con el lenguaje sexista en el marco universitario es un factor clave para avanzar en la Igualdad y la prevención de la Violencia de género el estudiantado no manifiesta, en la misma medida, la necesidad de usar un lenguaje inclusivo para no discriminar (UV:32\% y UAEM: 43\%). Es una discrepanciaclara entre las declaraciones formuladas y las conductas a realizar. 


\section{Consideraciones finales}

A pesar de las interpretaciones erróneas, viciadas, contaminadas y mal realizadas (con y sin propósito) que se han hecho y hacen del feminismo una cosa está clara: hemos de abrazarlo con fuerza y que impregne nuestro saber ser. Más concretamente, en el ámbito universitario, nuestro saber (con)vivir. Ser feminista, postularse y actuar como tal, es una decisión sociopolítica en detrimento del heteropatriarcado, en favor de la justicia y el bienestar social de todos, pero en especial de todas. Las Universidades han de ser un marco que acoja y desarrolle estos principios. De lo contrario, estamos faltando severamente al compromiso con ese tesoro que encierra la Educación de la que nos habla Delors; pero, además, estamos atentando brutalmente contra el derecho a ser, contra la dignidad de muchas mujeres por intereses, valores y creencias totalmente arbitrarios, también recogidos en numerosas cartas magnas y en la Declaración Universal de Derechos Humanos.

Acercarse a las y los demás haciendo el ejercicio, hermoso y gigante, de (RE)conocer su otredad es algo que debe inspirarnos a todas y todos. No hablamos (al menos, no solo) de formación integral y específica desde la docencia para la discencia, sino de una educación cívica, moral, en valores que nos preocupa y ocupa a todo el equipo universitario, sin rangos, títulos o distinción alguna; el feminismo, liderado por ellas, es cosa de todas, pero también de todos. En muchas ocasiones, basta con practicar la escucha activa, tomar consciencia de nuestros privilegios (por cuestión de clase, etnia o, como en este caso, género) e ir renunciando a ellos paulatinamente, arrimando el hombro para que el resto del mundo pueda también liberarse y liberar del yugo de esta opresión tan antigua.

Tornemos las Universidades en espacios seguros, en espacios feministas.

\section{Referencias}

Bell, A. y Smith, D. (2001). Aprenda a tratar con personas conflictivas. Barcelona, España: Editorial Gestión 2000.

Butler, J. (1990). El género en disputa. Madrid: Paidós.

Cadavid, T.E. (2009). Sobre la universidad-empresa. Revista Iberoamericana De Educación, 50(7), pp. 1-4. Recuperado de https://rieoei.org/RIE/article/view/1952

Cobo, R. (2009). Otro recorrido por las Ciencias Sociales: género y teoría crítica. En Aparicio, M.; Leyra, B. y Ortega, R. (Ed.) (2009). Cuadernos de género: Políticas y acciones de género. Materiales de formación (pp. 11-52). Madrid: Universidad Complutense.

Cortina, A. (2013). ¿Para qué sirve realmente...? La ética. Barcelona, España: Paidós

De Bono, E. (1985). Conflictos: cómo resolverlos de la mejor manera. Barcelona, España: Plaza \& Janés.

Delors, J.,Al mufti, I., Amagi, I., Carneiro, R., Chung, F., Geremek, B., Gorham, W., Kornhauser, A., Manley, M., Padrón quero, M., Savane, mM-A., Singh, K., Stavenhagen, R., Myong 
won suhr y Zhou nanzhao, Z. La educación encierra un tesoro. Informe a la UNESCO de la Comisión Internacional sobre la Educación para el Siglo XXI. París: UNESCO, 1996

De Miguel, A. (2007). El proceso de redefinición de la violencia contra las mujeres: de drama personal a problema político.Daimon. Revista de Filosofía, 42, pp. 71-82.

Enciso, V. y Rico, A. (2009). Las y los académicos de la Universidad de Guadalajara: una aproximación de género. En M. Chávez et al. (coord.) (2009). Género y trabajo en las universidades (pp. 309-339). México: Instituto Municipal de las Mujeres en Guadalajara.

Fontanela, M. (2008). Diccionario de estudios de Género y Feminismos. Buenos aires: Editorial Biblos.

Ferrer, V., Bosch, E., Ramis, M. y Torres, G. (2006). La violencia contra las mujeres en la pareja: creencias y actitudes en estudiantes universitarios. Psicothema, 18 (3), 359-366.

Goldman, E. (2017). Feminismo y anarquismo. Enclave.

Hooks, b. (1990). Yearning: Race, Gender, and Cultural Politics.

Luxán Serrano, M., Imaz Bengotxea, J., Bereziartua Etxeberria, G., y Lauzurika Arrondo, A. (2014). Universidad y Transformación Social. Revista De La Asociación De Sociología De La Educación (RASE), (7).

Macías, J.; Gil, E.; Rodríguez, Mª A.; González, J.R.; González, Mª M. y Solter, A. Ma (2012). Creencias y actitudes del estudiantado de Enfermería sobre la Violencia de Género. Index Enfermería, 21 (1-2), 1-8.

Ministerio de sanidad, servicios sociales e igualdad. secretaría de igualdad (2011), MacroencuestadeViolenciadeGénero2011.Disponibleen: http://www.observatorioviolencia.org/upload_images/File/DOC1329745747_macroencue sta2011_principales_resultados-1.pdf.

Ministerio de sanidad, servicios sociales e igualdad. secretaría de igualdad (2015), Percepción Social de la Violencia de Género.Disponible en: http://www.violenciagenero.msssi.gob.es/violenciaEnCifras/estudios/colecciones/pdf/Perc epcion_Social_VG_web.pdf

Mujeres Para la Salud. (2018). Informe: Efectos y consecuencias de la violencia y el maltrato doméstico a mujeres - Mujeres para la Salud. Disponible en https://www.mujeresparalasalud.org/informe-efectos-y-consecuencias-de-la-violencia-yel-maltrato-domestico-a-mujeres/

Observatorio de Igualdad de Género de América Latina y el Caribe (2015). Informe Anual 20132016. El enfrentamiento de la violencia contra las mujeres en América Latina y el Caribe. Disponible en https://repositorio.cepal.org/bitstream/handle/11362/37185/S1500499_es.pdf?sequence=4\&isAllo wed $=\mathrm{y}$ 
Organización Naciones Unidas (2017. ONU Mujeres. Informe 2016-2017. Disponible en http://www.unwomen.org/-

/media/headquarters/attachments/sections/library/publications/2017/un-women-annualreport-2016-2017-es.pdf?la=es\&vs=1453.

Palomar, C. (2009). La perspectiva de género en la Universidad de Guadalajara. En Marum, E. (2009): Liderazgo y equidad. Una perspectiva de género. México: UdG (pp.231- 243). Disponible

en: http://cumle.cucea.udg.mx/Documentos/Libros/Liderazgo \%20y \%20equidad.pdf

Palomar, C. (2011). La cultura institucional de género en la Universidad de Guadalajara. México: ANUIES.

Ramírez, G. (2008). Diagnóstico sobre la violencia en la educación media superior y las relaciones entre estudiantes y docentes para la promoción de los derechos humanos y las relaciones igualitarias. México: Academia Mexicana de Derechos Humanos, AC.

Silva, R. (2015). Actualidad Política y Cultural, ¿en que consiste el modelo heteropatriarcal? Disponible en http://rafaelsilva.over-blog.es/2015/09/en-que-consiste-el-modeloheteropatriarcal-i.html.

Solà, M., y Urko, E. (2013). Transfeminismos. Txalaparta.

Varela, N. (2005). Feminismo para principiantes. Barcelona, España: Ediciones B.

Viana, I. (2012). Mediación escolar y observatorios para la convivencia. Estudio comparado entre Comunidades Autónomas. Cuestiones pedagógicas, 21, 229-248.

Wittig (2006). El pensamiento heterosexual. Barcelona, España: Egales. 